Original Paper http://ajol.info/index.php/ijbcs http://indexmedicus.afro.who.int

\title{
Etude de la dynamique de Bactrocera dorsalis (Hendel) (Diptera: Tephritidae) dans les vergers de mangues en Basse Casamance : influence des facteurs climatiques
}

\author{
Ibrahima Solo KONTA ${ }^{1 *}$, Saliou DJIBA ${ }^{2}$, Seyni SANE ${ }^{1}$, Landing DIASSI ${ }^{2}$, \\ Abdoulaye Baila NDIAYE ${ }^{3}$ et Kandioura NOBA ${ }^{1}$ \\ ${ }^{I}$ Département de Biologie Végétale, Faculté des Sciences et Techniques, Université Cheikh Anta Diop de \\ Dakar, B. P. 5005, Dakar Fann, Sénégal. \\ ${ }^{2}$ ISRA/CRA de Djibélor route du Cap Skirring B.P 34 Ziguinchor, Sénégal. \\ ${ }^{3}$ Laboratoire de Zoologie des Invertébrés Terrestres, Institut Fondamental d'Afrique Noire (IFAN), UCAD, \\ B. P. 206, Dakar, Sénégal. \\ *Auteur correspondant, E-mail: ibrahimasolo2002@yahoo.fr ; Tel: +221 786371631
}

\section{REMERCIEMENTS}

Nous adressons des remerciements au Ministère de l'Enseignement Supérieur, des Universités et des Centres Universitaires Régionaux et de la Recherche Scientifique pour le soutien financier dans l'accomplissement de ces travaux.

\section{RESUME}

Au Sénégal, la filière mangue contribue à la lutte contre la pauvreté et l'insécurité alimentaire en milieu rural. Toutefois, elle est confrontée à des contraintes phytosanitaires notamment celles liées à Bactrocera dorsalis. La présente étude qui vise à comprendre la dynamique $B$. dorsalis a consisté à placer dans 4 vergers de manguiers, des pièges de type Sentrap contenant un diffuseur d'un attractif à base de méthyl eugénol et une plaquette d'un insecticide, Dichlorvos et à relever et dénombrer les captures. Les résultats montrent que $B$. dorsalis est présente à des degrés différents dans les vergers étudiés toute l'année. Le nombre de mouches capturées est plus élevé en première année de piégeage (1.547 individus en moyen) et faible en deuxième année (743 individus en moyen). Les captures de saison des pluies sont plus importantes que celles de saison sèche. La charge en fruit des plantes-hôtes et l'hygrométrie sont apparues comme deux facteurs majeurs influençant les fluctuations des populations de mouches. Une corrélation positive est observée entre la dynamique, la température, l'hygrométrie et la pluviométrie mais négative entre la dynamique et le vent. Les périodes de faibles «densités» semblent être les périodes les plus propices pour le contrôle des mouches.

(c) 2015 International Formulae Group. All rights reserved.

Mots clés: Bactrocera dorsalis, dynamique, facteurs climatiques, piégeage.

\section{Study of the dynamics of Bactrocera dorsalis (Hendel) (Diptera: Tephritidae) in mango orchards in Basse Casamance: influence of climatic factors}

\section{ABSTRACT}

In Senegal, the mango industry contributes to the fight against poverty and food insecurity in rural areas. However, it faces phytosanitary constraints including those related to Bactrocera dorsalis. This study, aimed at understanding the dynamics of $B$. dorsalis, was conducted in four mango orchards, using Sentrap type 
of traps, each containing an attractive (methyl eugenol) diffuser and insecticide (dichlorvos) plate. The catch of every trap is collected, sorted out and counted by specie. The results show that $B$. dorsalis is present, to varying degrees, in the studied orchards throughout the year. The number of flies captured is higher in the first year of trapping (1.547 flies in average) and low in the second year (743 individuals in average). The rainy season catches are larger than those of the dry season. The host plants fruit load and humidity appeared to be two major factors influencing the fluctuations in fly populations. A positive correlation was observed between the dynamics, temperature, humidity and rainfall. But a negative correlation was noted between fly population dynamics and wind. Periods of low "densities" seem to be the most favorable periods for fly control.

(c) 2015 International Formulae Group. All rights reserved.

Keywords: Bactrocera dorsalis, dynamic, climatic factors, trapping.

\section{INTRODUCTION}

Le Sénégal bénéficie de conditions climatiques et pédologiques favorables à la production horticole (Asepex, 2012). L'horticulture est pratiquée dans toutes les régions du pays et constitue une des premières activités de production rémunératrice en milieu rural. Elle est également l'une des filières les plus dynamiques du secteur agricole en raison de la permanence de ses activités dans certaines zones, du nombre de pratiquants au niveau national, de la diversité des espèces cultivées et des régions touchées (Asepex, 2012). De toutes les filières, la mangue est celle qui contribue le plus largement à la stratégie de lutte contre la pauvreté et l'insécurité alimentaire. En effet, elle constitue un apport nutritionnel fondamental pour les populations rurales en période de soudure (USDA, 2001).

Depuis l'invasion de la mouche Bactrocera dorsalis (Vayssière et al., 2004), la filière mangue enregistre des contreperformances malgré les investissements consentis par l'État du Sénégal (Ndiaye, 2008) et le dynamisme du secteur privé dans le développement. Très peu de recherches ont été menées sur les mouches des fruits au Sénégal. Avant l'invasion de $B$. dorsalis, les premières études réalisées dans ce domaine concernaient les espèces indigènes (Cératites). Après 2004, quelques travaux sont notés sur les plantes hôtes de $B$. dorsalis (Mané, 2008), sur l'évaluation de la dynamique de populations de $B$. dorsalis (Sonkho, 2010), sur le piégeage des mouches des fruits (Ndiaye et Leussieur, 2012) et sur les plantes hôtes et parasitoïdes de la mouche des fruits (Ndiaye, 2014). Toutefois, très peu d'études ont porté sur la bio écologie des espèces et sur la dynamique des populations. Or, la connaissance des cycles de développement des mouches, des plantes hôtes et des mécanismes contrôlant la dynamique des populations, est essentielle dans la réussite des différentes méthodes de gestion des ravageurs. Il s'avère donc important de bien cerner les périodes de forte et faible population de ces ravageurs en fonction de la variabilité des stades phénologiques des fruits hôtes, de la pluviométrie et de la température pour une meilleure gestion des populations de mouches des fruits.

Ce travail vise à étudier la dynamique de $B$. dorsalis, un ravageur invasif et dévastateur, en vue de proposer des méthodes de gestions efficaces et durables. Il cherche plus spécifiquement à déterminer les périodes de fortes et de faibles populations au cours de l'année et à étudier l'influence des facteurs climatiques sur la dynamique de la population de B. dorsalis.

\section{MATERIEL ET METHODES \\ Zones d'étude \\ Position géographique}

Notre zone d'étude, la région de Ziguinchor ou la Basse Casamance, est située au Sud-Ouest du Sénégal entre les latitudes $12^{\circ} 20$ et $13^{\circ}$ et les longitudes $16^{\circ}$ et $16^{\circ} 50$ (Figure 1). Elle couvre une superficie de 7,339 $\mathrm{km}^{2}$, soit $3,74 \%$ de l'ensemble du territoire national. Elle est limitée au Nord par la Gambie, au Sud par la Guinée Bissau, à l'Est par la région de Sédhiou et à l'Ouest par l'Océan Atlantique (Ba, 2004). 


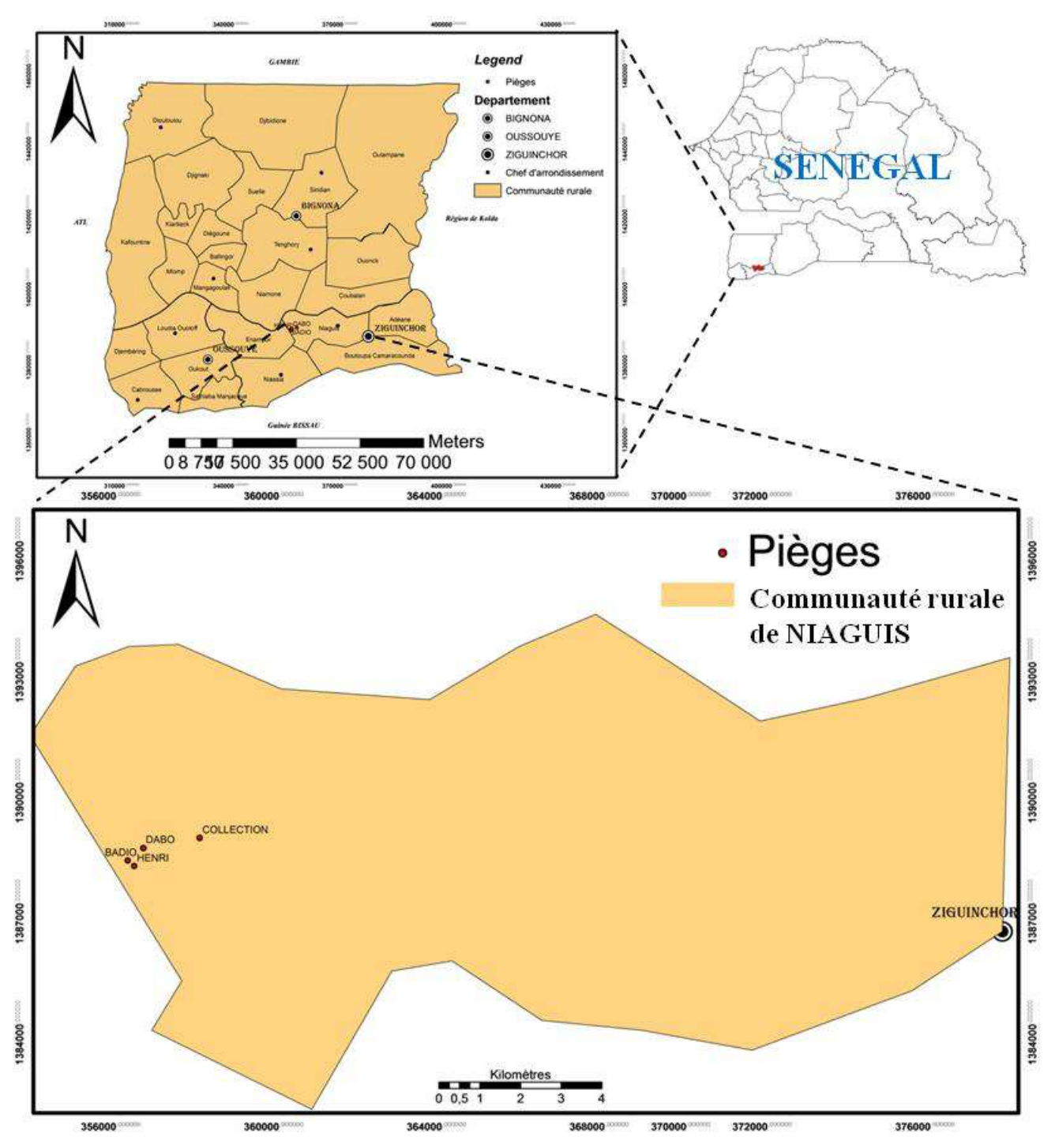

Figure 1: Carte de la zone d'étude et position des pièges de collecte.

\section{Potentialité de la zone d'étude}

La Base Casamance est une région écologiquement favorable pour le développement de l'arboriculture fruitière : la pluviométrie est relativement bonne (de 700 à plus de $1200 \mathrm{~mm}$ par an) et les sols assez diversifiés. La mangue casamançaise a un avantage comparatif par rapport à sa disponibilité sur les marchés national, sous régional et international. En effet, sur le plan intérieur, elle est sur le marché avant les mangues des autres régions productrices, et sur le marché international, après les mangues du Mali, de la Côte d'Ivoire et du Burkina Faso et avant la mangue de l'Israël. Cet avantage écologique dont bénéficie la Casamance lui confère la place de leader sur le marché international pendant la période allant de mi-juin à fin juillet.

\section{Sites de collecte}

Les critères de choix des sites reposent sur le nombre important de variétés d'arbres 
fruitiers précoces et tardives ; l'accessibilité, la taille du verger qui ne devrait pas mesurer moins d'un (1) ha et la collaboration et l'engagement des planteurs à ne pas perturber les piégeages sous aucune forme.

Sur cette base, 4 sites ont été choisis et un piège a été posé par site (Figure 1) : site1 (collection ISRA, bien entretenu), site 2 (Badio, moins entretenu), site 3 (Henry, bien entretenu) et site 4 (Dabo, verger presque abandonné). Afin d'éliminer les éventuelles interactions entre les pièges, la distance minimale entre deux vergers est fixée à 120 $\mathrm{m}$.

Etude de la dynamique de la population par piégeage des mouches adultes des fruits

Ce piégeage a pour objectif de mesurer dans le temps l'évolution de la population de B. dorsalis. Des pièges de type Sentrap ont été placés dans des vergers en vue de suivre l'évolution de la population de ce ravageur. La cage Sentrap est une bouteille vide en plastique munie dans sa partie supérieure d'un orifice servant d'entrée pour les mouches. Elle est colorée partiellement en jaune. La couleur jaune est attractive pour la mouche gravide. A l'intérieur de chacune des cages sont introduits deux comprimés: l'un à base d'insecticide (Dichlorvos DDVP, Vapona : 2,2-Dichlorovinyl dimethyl phosphate) et l'autre à base d'attractif sexuel (méthyl eugenol) de mouches des fruits spécifique à $B$. dorsalis.

Un piège est placé par site de juillet 2012 à juillet 2014 et la collecte des captures se fait toutes les semaines. L'attractif et l'insecticide sont renouvelés une fois par mois. Les spécimens capturés dans les pièges sont comptés puis conservés dans de l'éthanol à $70^{\circ}$ pour identification.

\section{Etude de l'influence des facteurs climatiques}

L'influence des facteurs climatiques (pluviométrie, température, vitesse du vent et humidité relative de l'air) sur la dynamique de la mouche des fruits dans les vergers a été étudiée. Les instruments de mesure des facteurs climatiques qui ont été utilisés sont :

le pluviomètre pour les quantités de pluies tombées;

- l'anémomètre pour la vitesse du vent ;

le psychromètre pour l'humidité relative et la température de l'air.

Les relevés ont été fait 24 heures sur 24 heurs à $50 \mathrm{mn}$ de chaque heures.

Les données climatiques ont été fournies par le service météo de Ziguinchor situé à $3 \mathrm{~km}$ environ des sites de piégeages.

\section{Traitement de données}

La saisie et le traitement des données ont été effectués grâce au tableur Excel et les analyses statistiques grâce au logiciel SigmaStat (version 2.0, Jandel Corporation, 19921995). Du fait de la grande variabilité des données, nous avions effectué une transformation en $\log 10$ pour écraser les écarts trop élevés entre les données. Ces différentes données ont été analysées statistiquement en adoptant une approche paramétrique. Les comparaisons ont été faites grâce au test d'analyse de variance (ANOVA à deux ou trois facteurs) suivies du test de Student Newman-Keuls quand les différences étaient significatives. Une régression linéaire multiple a été effectuée pour mettre en évidence l'influence des facteurs climatiques sur les captures au cours de la période d'étude grâce au logiciel R (version R-3.1.2, package ADE4), R Development Core Team (2004). Dans tous les cas, le seuil de significativité était fixé à $5 \%$.

\section{RESULTATS}

Dynamique de la population de Bactrocera dorsalis dans la zone d'étude

Les résultats de captures par site de piégeage de juillet 2012 à 2014 sont présentés dans le Tableau 1. Au total, 123.597 individus de Bactrocera dorsalis ont été capturés au cours de cette étude dans les quatre sites étudiés. Les captures les plus élevées ont été obtenues dans le site 4 , soit 43.507 mouches et le plus faible dans le site 1 , soit 20.437 
mouches. Les pourcentages de mouches capturées sont plus importants dans les sites 4 $(35.2 \%)$ et $2(29.4 \%)$ et plus faibles dans le site $3(18.9 \%)$ et $1(16.5 \%)$. L'observation des résultats des captures mensuelles de $B$. dorsalis entre juillet 2012 et juillet 2014 montre de façon générale que les captures les plus faibles sont observées en décembre, à l'exception du site 4, et en mars et augmentent de mai à juillet. En 2012, les captures ont été nettement plus élevées au mois de juillet 2012 dans les 4 sites que tous les autres mois sur les deux ans. Puis en 2013, les captures sont plus élevées en juillet dans les sites 1 et 3 et en août dans les sites 2 et 4 . Et en fin en 2014, les captures sont plus importantes en juillet dans les sites 1, 2 et 3 et en juin dans le site 4; elles sont faibles dans tous les sites en janvier, février et mars.

Une évolution cyclique est observée chaque année avec des captures relativement plus importantes entre les mois de mai et septembre dans les quatre sites et au cours de d'étude.

\section{Evolution inter annuelle des captures}

La Figure 2 montre les variations de mouches capturées aux cours des différentes années de piégeages. Le nombre moyen de mouches capturées varie d'une année à une autre. En 2012, le nombre est de 515 en moyenne contre 139 en 2013 et 239 en 2014. L'analyse statistique montre que les captures sont significativement plus élevées en 2012 comparées aux autres années (2013 et 2014), $(\mathrm{p}<0.001)$. Les captures de 2014 sont légèrement plus élevées que celles de 2013. Par contre, aucune différence significative n'est observée entre ces deux années au seuil de $5 \%$.

\section{Evolution inter annuelle des captures par} sites

La Figure 3 présente l'évolution des captures en fonction des sites et des années. Une variabilité des captures est observée entre les sites et au cours des trois années.

En 2012, les captures les plus élevées sont obtenues dans le site $2(780,76)$ et les plus faibles dans le site $1(56,66)$. En 2013, les captures les plus élevées sont observées dans les sites 1 et 4 respectivement (301,61 et 283) et les plus faibles dans les sites 3 et 2 respectivement $(15,89$ et 41,24$)$. En 2014 , les captures les plus élevées sont obtenues dans les sites 4, 2 et 1 respectivement $(317,93$, $299,92$ et 256,95$)$ et le plus faible dans le site 3 (84,78). L'année 2012 est caractérisée par les captures plus élevées avec un cumul de 2060.14 comparé aux autres années 2014 $(959,58)$ et $2013 \quad(641,75)$. Cependant, l'analyse statistique montre qu'il n'existe pas d'interaction significative au seuil de $5 \%$ entre les captures de sites par rapport aux années $(\mathrm{p}=0,90)$.

\section{Evolution saisonnière des captures par années}

La Figure 4 présente les interactions entre les captures des saisons par rapport aux années. Les captures sont plus élevées en saison humide qu'en saison sèche pour toutes les trois années avec des valeurs moyennes respectives de 585,74 (2012) ; 210,52 (2013) et 310,60 (2014). Les plus faibles captures sont obtenues en 2013 comparées aux années 2012 et 2014. Les captures, selon les saisons, sont significativement plus importantes en 2012 comparés aux années 2013 et 1014 ( $p<0.001)$. Cependant, aucune différence statistique significative des captures au seuil de 5\% n'est observée entre 2013 et 2014.

\section{Evolution saisonnière des captures par sites}

La Figure 5 présente les captures par saisons selon les sites. Pour chacun des sites, les captures de saison humide sont légèrement plus importantes que les captures de saison sèche. Les captures par saisons des sites 1 et 4 respectivement 461,17 (saison humide) et 427,57 (saison sèche), et 527,19 (saison humide) et 493,52 (saison sèche) sont plus importantes que les captures par saisons des sites 2 et 3 (humide : 85,95; sèche : 52,28 et humide : 186,03 ; sèche: 152,36). Cependant, les résultats de l'analyse statistique montrent qu'il n'existe pas de différence significative 
au seuil $5 \%$ entre ces captures de saisons par sites $(\mathrm{p}=0,10)$.

\section{Influence des facteurs climatiques}

Le Tableau 2 présente la matrice des coefficients de corrélation (R) entre les captures des sites et les paramètres climatiques. L'analyse des facteurs climatiques et le nombre de mouches des fruits capturées ont montré que les captures de mouches des fruits étaient corrélées avec tous les facteurs abiotiques notamment la pluviométrie, l'humidité relative et la température (coefficient de corrélation R : 0,4$0,7)$ sauf avec la vitesse du vent (coefficient de corrélation $\mathrm{R}$ : [-0,2]-[-0,4]).

\section{Influence de la température de l'air sur l'évolution des captures}

La Figure 6 traduit l'évolution des captures mensuelles en relation avec les variations mensuelles de la température. Elle montre que l'évolution des captures suit légèrement celles de la température. Les fortes captures sont constatées durant les périodes chaudes allant de mai à novembre et les faibles captures pendant les périodes fraîches. Globalement, il y a une évolution saisonnière de la température et des captures au cours de l'étude. Une corrélation positive et significative est observée entre les captures par site et la température de l'air (Tableau 2, Pearson Correlation, $p<0.05$ ).

Influence de l'humidité de l'air sur l'évolution des captures

La Figure 7 traduit la dynamique de Bactrocera dorsalis en relation avec l'humidité de l'air. La dynamique de Bactrocera dorsalis présente une grande variabilité dans le temps. Elle est corrélée avec l'humidité relative de l'air. Les fortes captures sont observées lorsque l'humidité de l'air est importante allant de la période de mai à novembre avec 118,069 mouches capturées. Les faibles captures sont observées de décembre à avril coïncidant à des humidités relativement basses avec 5,528 mouches collectées. Globalement il y a une évolution saisonnière de l'humidité de l'air et du nombre de mouches capturées au cours de cette étude. Une corrélation positive et significative est observée entre les captures par site et l'humidité de l'air (Tableau 2, Pearson Correlation, $p<0.05$ ).

Influence de la pluviométrie sur l'évolution des captures

La Figure 8 présente la dynamique de Bactrocera dorsalis dans la zone d'étude en relation avec la pluviométrie. Le nombre de mouches capturées dans les pièges varie en fonction de la pluviométrie. Les captures les plus élevées sont constatées durant les périodes pluvieuses dans la zone. Globalement, sur les deux années de piégeage, les captures ont été plus importantes à partir de mai à novembre dans les vergers. Une corrélation positive et significative est observée entre les captures par site et la pluviométrie (Tableau 2, Pearson Correlation, $p<0.05)$.

Influence de la vitesse du vent sur l'évolution des captures

La Figure 9 traduit l'évolution des captures en relation avec la vitesse du vent. Elle montre que les fortes captures sont observées lorsque la vitesse du vent est faible. Une corrélation négative non significative est observée entre les captures par site et la vitesse du vent (Tableau 2, Pearson Correlation, $p>0.05)$.

Coefficients du modèle de la régression des captures par les facteurs abiotiques

La régression multiple effectuée entre les captures d'insectes (log du nombre des insectes de mouches des fruits capturés) et les facteurs climatiques montre que l'humidité relative est le facteur qui permet d'expliquer le plus les captures au cours de la période d'étude (Tableau 3). Elle augmente de manière significative les captures d'insectes avec un coefficient de 0,05 par rapport au log du nombre d'insectes capturés. 


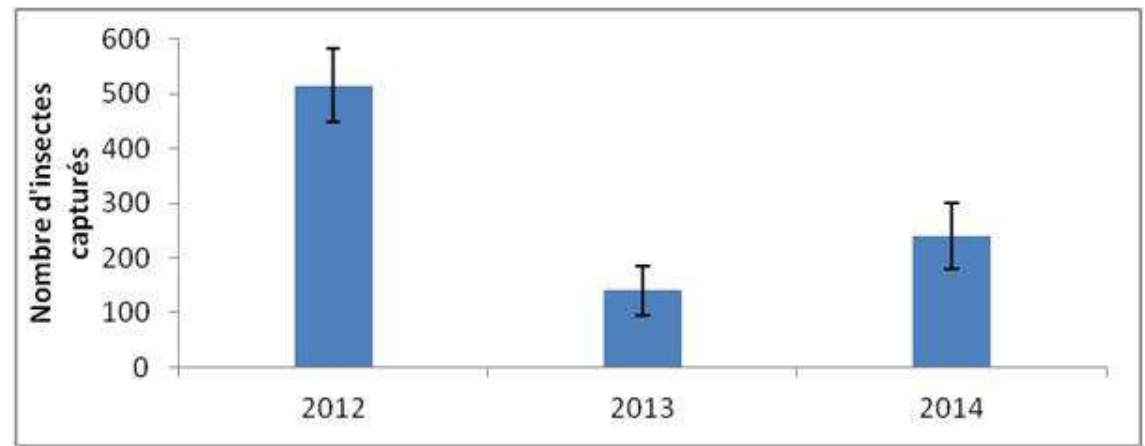

Figure 2 : Répartition des captures moyennes selon les années.

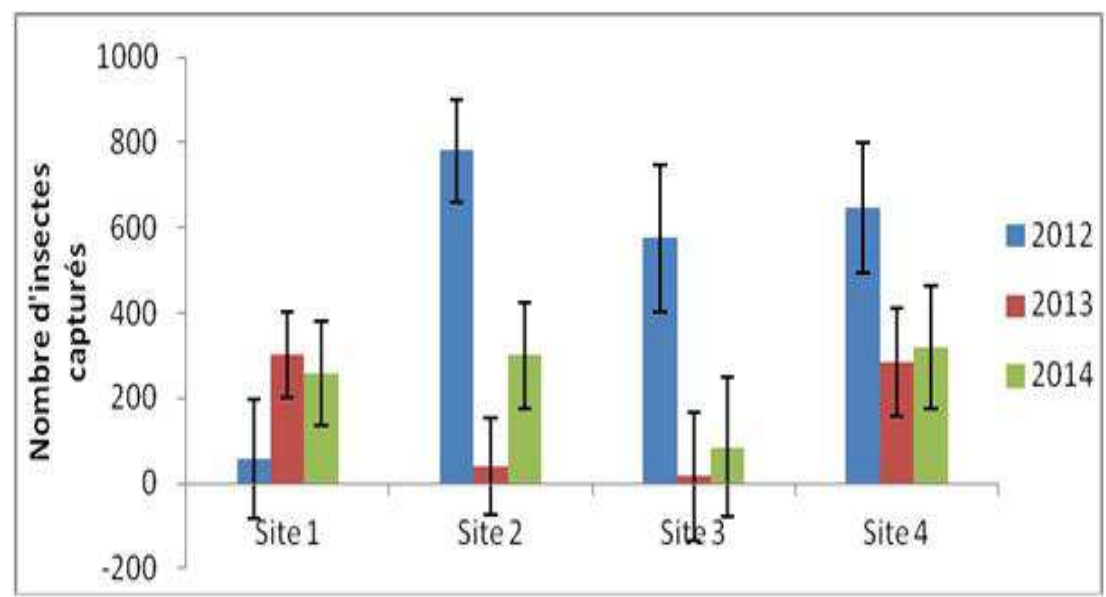

Figure 3 : Répartition des captures moyennes des sites par rapport aux années de piégeage.

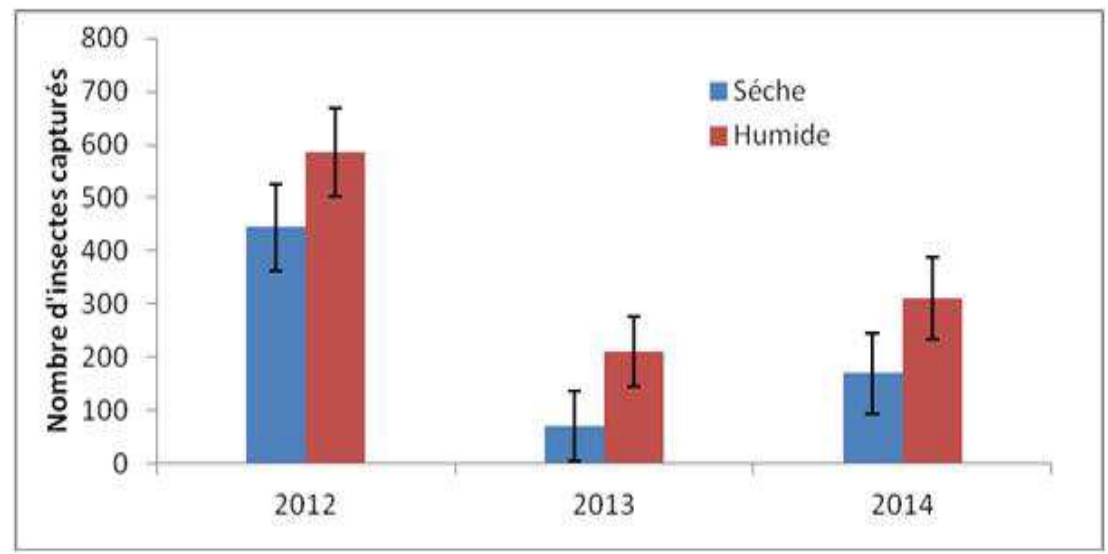

Figure 4 : Répartition des captures moyennes par année selon les saisons. 


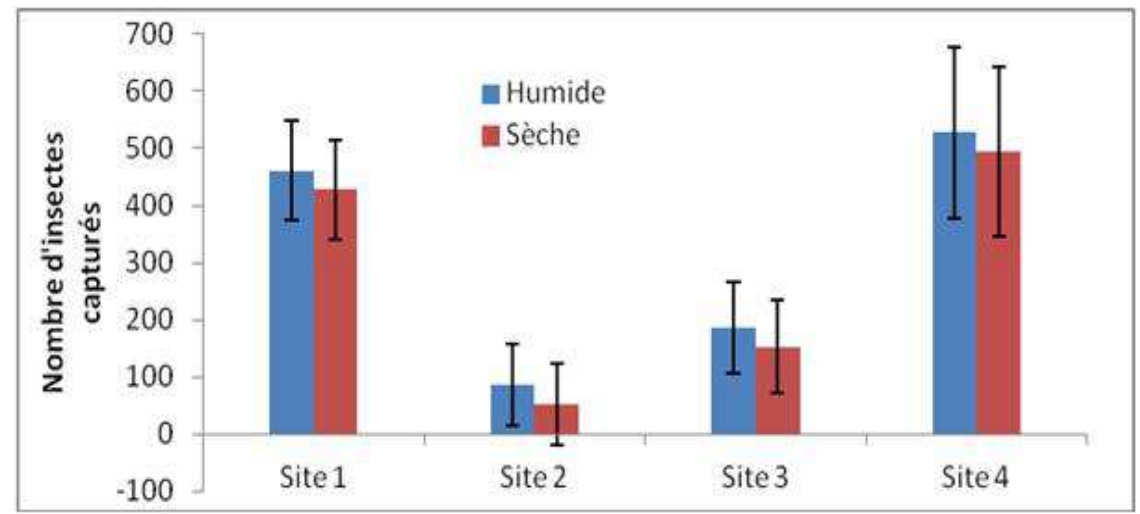

Figure 5 : Répartition des captures moyennes des saisons selon les sites d'étude.

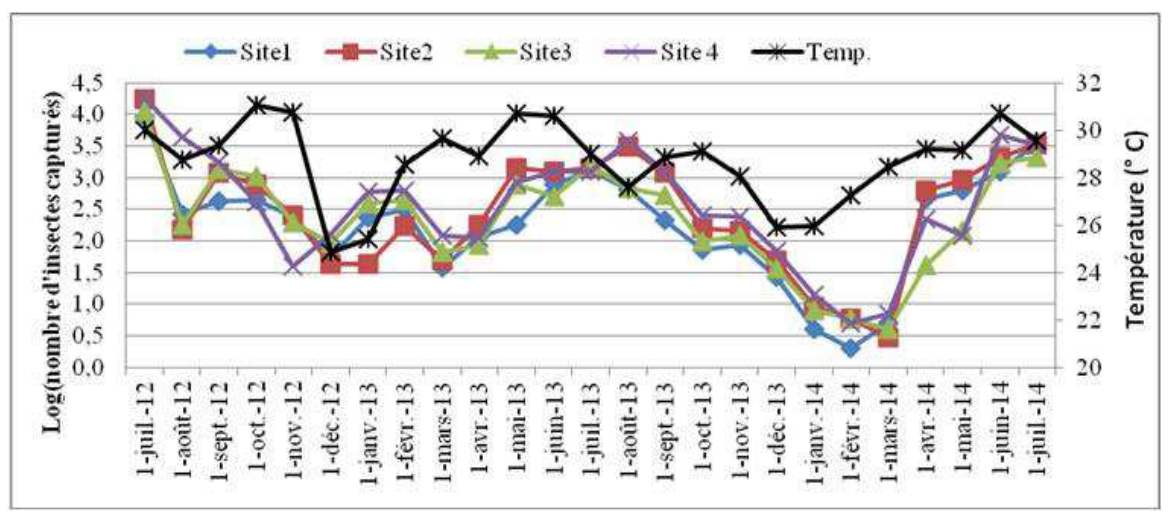

Figure 6 : Température de l'air et évolution des captures.

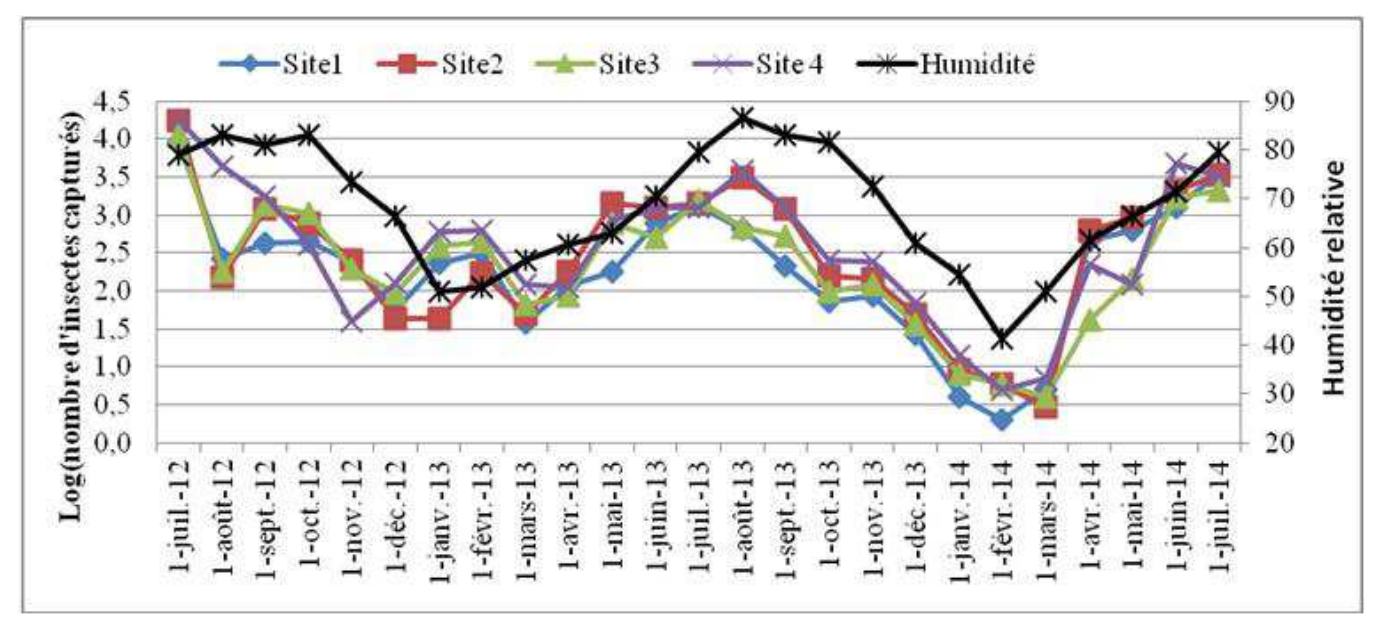

Figure 7 : Humidité de l'air (hygrométrie) et évolution des captures. 


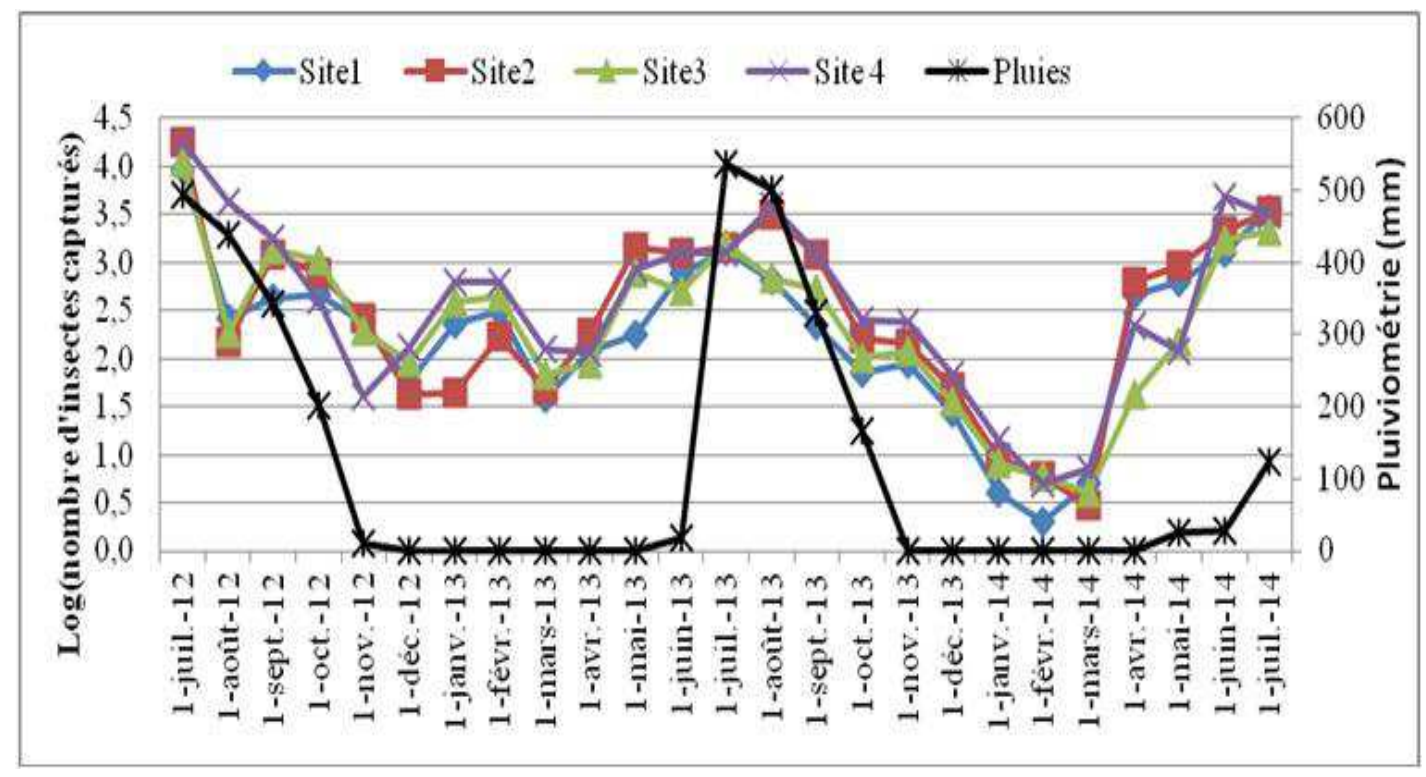

Figure 8 : Pluviométrie et évolution des captures.

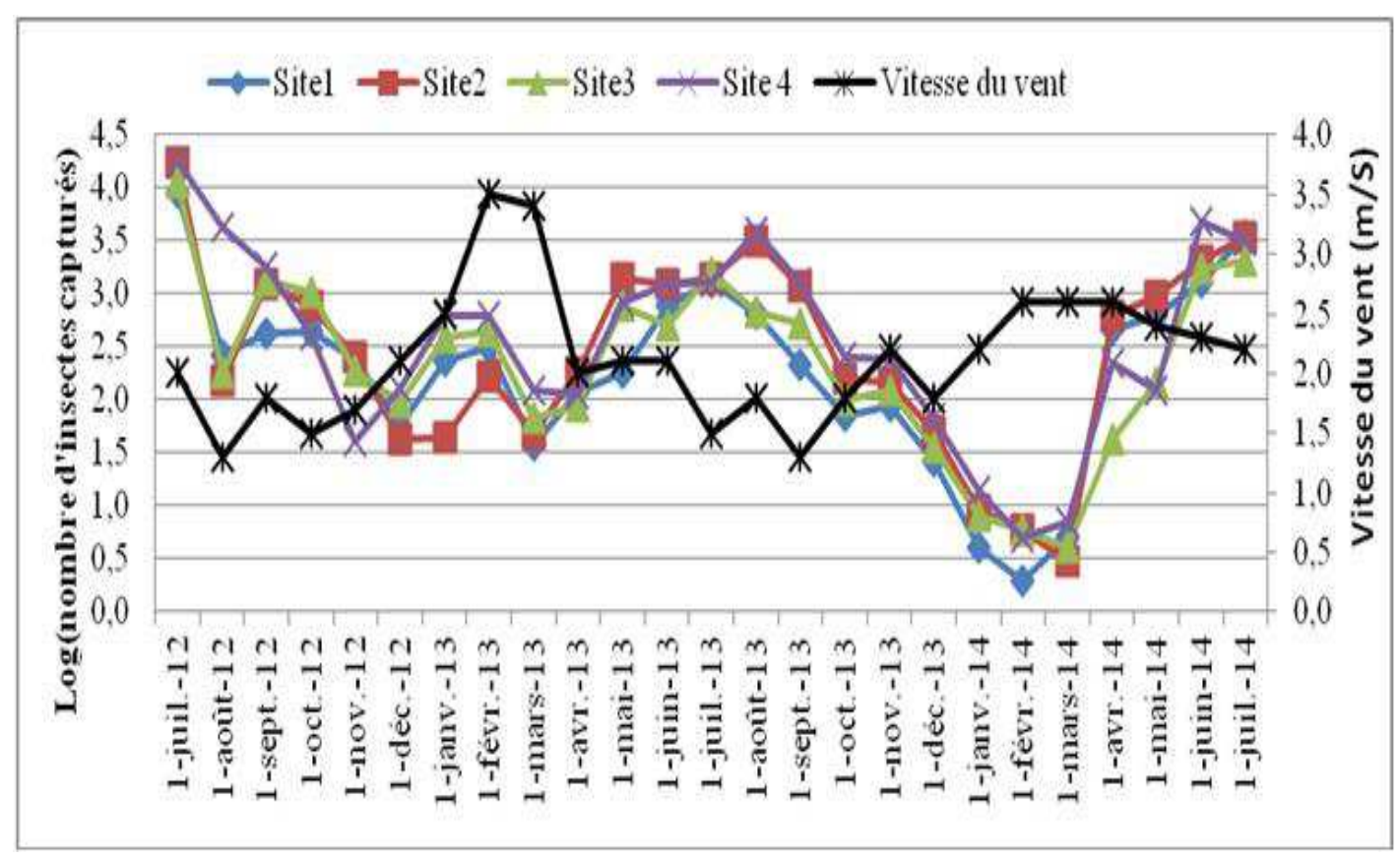

Figure 9 : Vitesse du vent et évolution des captures. 
I. S. KONTA et al. / Int. J. Biol. Chem. Sci. 9(6): 2698-2715, 2015

Tableau 1 : Total mensuel et $\log 10$ des captures de Bactrocera dorsalis dans les quatre sites de la zone d'étude de 2012 à 2014.

\begin{tabular}{lcccccccc}
\hline Dates récoltes & Site1 & log10-site1 & Site2 & log10-site2 & Site3 & log10-site3 & Site 4 & log10-site4 \\
\hline juillet.-12 & 9070 & 3,96 & 17550 & 4,24 & 11183 & 4,05 & 17878 & 4,25 \\
août-12 & 261 & 2,42 & 151 & 2,18 & 181 & 2,26 & 4314 & 3,63 \\
sept.-12 & 422 & 2,63 & 1222 & 3,09 & 1362 & 3,13 & 1795 & 3,25 \\
oct.-12 & 451 & 2,65 & 779 & 2,89 & 1034 & 3,01 & 401 & 2,6 \\
nov.-12 & 243 & 2,39 & 257 & 2,41 & 192 & 2,28 & 40 & 1,6 \\
déc.-12 & 57 & 1,76 & 43 & 1,63 & 94 & 1,97 & 128 & 2,11 \\
janv.-13 & 231 & 2,36 & 44 & 1,64 & 393 & 2,59 & 613 & 2,79 \\
févr.-13 & 310 & 2,49 & 169 & 2,23 & 436 & 2,64 & 636 & 2,8 \\
mars-13 & 38 & 1,58 & 49 & 1,69 & 67 & 1,83 & 124 & 2,09 \\
avril.-13 & 118 & 2,07 & 183 & 2,26 & 87 & 1,94 & 113 & 2,05 \\
mai-13 & 178 & 2,25 & 1417 & 3,15 & 773 & 2,89 & 846 & 2,93 \\
juin-13 & 786 & 2,9 & 1248 & 3,1 & 505 & 2,7 & 1260 & 3,1 \\
juillet.-13 & 1316 & 3,12 & 1431 & 3,16 & 1571 & 3,2 & 1271 & 3,1 \\
août-13 & 650 & 2,81 & 3093 & 3,49 & 679 & 2,83 & 3905 & 3,59 \\
sept.-13 & 216 & 2,33 & 1183 & 3,07 & 519 & 2,72 & 1262 & 3,1 \\
oct.-13 & 72 & 1,86 & 160 & 2,2 & 100 & 2 & 252 & 2,4 \\
nov.-13 & 87 & 1,94 & 144 & 2,16 & 121 & 2,08 & 244 & 2,39 \\
déc.-13 & 27 & 1,43 & 50 & 1,7 & 36 & 1,56 & 69 & 1,84 \\
janv.-14 & 4 & 0,6 & 9 & 0,95 & 8 & 0,9 & 14 & 1,15 \\
févr.-14 & 2 & 0,3 & 6 & 0,78 & 6 & 0,78 & 5 & 0,7 \\
mars-14 & 5 & 0,7 & 3 & 0,48 & 4 & 0,6 & 7 & 0,85 \\
avril.-14 & 459 & 2,66 & 618 & 2,79 & 42 & 1,62 & 221 & 2,34 \\
mai-14 & 636 & 2,8 & 944 & 2,97 & 146 & 2,16 & 122 & 2,09 \\
juin-14 & 1277 & 3,11 & 2102 & 3,32 & 1708 & 3,23 & 4801 & 3,68 \\
juillet.-14 & 3521 & 3,55 & 3453 & 3,54 & 2098 & 3,32 & 3186 & 3,5 \\
Total par site & 20437 & 56,67 & 36308 & 61,12 & 23345 & 58,29 & 43507 & 63,93 \\
\% par site & 16,54 & & 29,38 & & 18,89 & & 35,2 & \\
\hline Cumul & Sites & & & & 123.597 & & & \\
\hline
\end{tabular}


Tableau 2. Matrice des coefficients de corrélation (R) entre les captures des sites et les paramètres climatiques.

\begin{tabular}{|c|c|c|c|c|c|}
\hline & Désignation & Site 1 & Site 2 & Site 3 & Site 4 \\
\hline \multirow{3}{*}{ Pluies } & $\mathrm{R}$ & 0.499 & 0.550 & 0.564 & 0.641 \\
\hline & P-value & 0.0111 & 0.00443 & 0.00334 & 0.000559 \\
\hline & Nombre Echantillon & 25 & 25 & 25 & 25 \\
\hline \multirow{3}{*}{ Température } & $\mathrm{R}$ & 0.522 & 0.598 & 0.485 & 0.372 \\
\hline & P-value & 0.00745 & 0.00161 & 0.0140 & 0.0669 \\
\hline & Nombre Echantillon & 25 & 25 & 25 & 25 \\
\hline \multirow{3}{*}{ Humidité } & $\mathrm{R}$ & 0.655 & 0.726 & 0.668 & 0.693 \\
\hline & P-value & 0.000380 & 0.0000401 & 0.000266 & 0.000122 \\
\hline & Nombre Echantillon & 25 & 25 & 25 & 25 \\
\hline \multirow{3}{*}{ Vitesse du vent } & $\mathrm{R}$ & -0.249 & -0.353 & -0.308 & -0.309 \\
\hline & P-value & 0.230 & 0.0833 & 0.134 & 0.133 \\
\hline & Nombre Echantillon & 25 & 25 & 25 & 25 \\
\hline
\end{tabular}

Tableau 3. Coefficients du modèle de la régression des captures par les facteurs abiotiques.

\begin{tabular}{lccccc}
\hline Variables & Coefficients & Std. Error & t value & $\operatorname{Pr}(>|t|)$ & Code \\
\hline Constante = Capture) & $-5,3587$ & 2,0416 & $-2,62$ & 0,016 & $*$ \\
Pluies & & & & & \\
Température & 0,0011 & 0,0009 & 1,24 & 0,230 & $*$ \\
Humidité & 0,1054 & 0,0774 & 1,36 & 0,188 &. \\
Vitesse du vent & $\mathbf{0 , 0 4 8 8}$ & 0,0181 & 2,69 & $\mathbf{0 , 0 1 4}$ & \\
\multicolumn{1}{c}{ Codes de significativité : "*** " 0,$001 ; " * *$ " 0,$01 ; " * * 0,05 ; " . " 0,1 ; " ~ " 1}$. & &
\end{tabular}

\section{DISCUSSION}

Dynamique de la population de $B$. dorsalis de juillet 2012 à juillet 2014 dans la zone d'étude

Les résultats de cette étude montrent que la population de $B$. dorsalis fluctue dans le temps et l'espace d'une période à une autre dans la zone d'étude. Ce résultat corrobore avec celui de Geurts et al. (2012) qui montrent que la fluctuation dans le temps et l'espace de B. dorsalis est liée à la disponibilité de l'hôte et à la variation des différences facteurs climatiques. Sutherst et Maywald (1985), Sutherst et al. (2007) rajoutent que la mouche des fruits notamment $B$. dorsalis traverse deux types de saison chaque année, une saison favorables à la croissance et une saison de stress au cours de laquelle la population diminue. Cette aptitude à surmonter les différents obstacles confère à cette espèce le caractère hautement concurrentiel et envahissant (Heger et Trepl, 2003). De façon générale, les captures les plus faibles sont observées en décembre, à l'exception du site 4 et en mars et augmentent considérablement de mai à juillet. Les faibles prises en décembre peuvent s'expliquer par l'absence de fruits hôtes servant de lieux de ponte de la femelle gravidie mais également par la baisse de température enregistrée ce mois. Cette période coïncide en effet à l'absence de fruits de mangues et à l'immaturité des fruits 
d'agrumes dans les vergers étudiés. Le même phénomène noté en mars est lié au fait par la récolte des fruits hôtes présents dans ces vergers, notamment d'agrumes, pendant que les mangues ne sont pas encore arrivées en maturité. Ces résultats corroborent les travaux de De Villiers et al. (2015), qui ont montré que la disponibilité et la phénologie de l'hôte joue un rôle dans la dynamique de la population de $B$. dorsalis. Ces auteurs rajoutent qu'en absence de fruits hôtes, aucune dynamique n'est observée même si les conditions abiotiques redeviennent favorables. Hussain et Ahmad (2015) ont montré également qu'en Erythrée, aucune infestation n'a été constatée sur les goyaves de novembre à janvier coïncidant avec les basses températures et une faible disponibilité de fruits-hôtes.

En 2012, les captures ont été nettement plus élevées au mois de juillet 2012 dans les 4 sites que tous les autres mois sur les deux ans. Deux facteurs semblent expliquer, en 2012, ce phénomène : la précocité de la pluie en 2012 et l'absence d'effet des parasitoïdes importés et lâchés en juillet 2012, coïncidant avec le début de l'expérimentation. Les premiers mois de lâchés ont certainement permis aux parasitoïdes de s'établir. L'effet de l'arrivée de la pluie précoce s'approche des résultats de Siebert et al. (2005). De Villiers et al. (2015) ont montré l'importance de l'irrigation dans le maintien et la dynamique de $B$. invadens dans les zones arides. Mwatawala et al. (2006a), Vayssières et al. (2008b; 2009d) ont rapporté que $B$. invadens est une espèce qui se développe mieux dans des conditions de fortes humidités. Magagula et al. (2015) rajoutent que les activités humaines telles que l'irrigation (dans le cas de l'horticulture ou autres), favorisent la distribution et l'installation de $B$. dorsalis même dans les zones peu richesse en plantes-hôtes.

En 2013, les captures ont été plus élevées en juillet dans les sites 1 et 3 et en août dans les sites 2 et 4 . Le facteur qui semble expliquer les plus fortes captures en juillet 2013 dans le 1 et 3 et en août dans le site 2 et 4 serait lié à l'hétérogénéité des vergers du point de vue sanitaire. Les vergers les plus entretenus ont des niveaux de captures précocement plus élevés que les parcelles les moins entretenues. Ces vergers présentement également plus de variétés précoces.

En 2014, les niveaux de captures précocement plus élevés dans les sites 2 et 4 comparés à ceux obtenus en 2013 sont dus au fait que ces parcelles ont été entretenus au cours de cette année. En plus, il pourrait s'agir d'une alternance de production généralement observée en arboriculture fruitière favorisant une plus grande disponibilité des fruits de mangues en 2014.

La quasi absence de prise notée entre janvier et mars 2014 s'explique par la non disponibilité de fruits de mangues et d'agrumes.

L'analyse des résultats obtenus du nombre moyen de mouches capturées par années montre que les captures sont 2 à 3 fois plus élevées en 2012 qu'en 2013 et 2014. Entre 2013 et 2014, les prises sont plus importantes en 2014 qu'en 2013. Les parasitoïdes lâchés en 2012 ont certainement agi plus efficacement en 2013 et ont vu leur action diminuer en raison probablement de la mortalité progressive ou de la concurrence avec les parasitoïdes locaux en 2014.

Ainsi, quelle que soit l'année, les captures qui sont plus élevées en saison humide qu'en saison sèche, trouvent leur explication dans le fait qu'il $y$ a une disponibilité et une diversité de fruits de mangues et de fruits sauvages correspondant à leur réceptivité allant de mai à octobre. En plus, les conditions climatiques sont nettement plus propices au développement de la mouche des fruits dans la zone d'étude en cette période. Ces résultats concordent avec celui de Guerts et al. (2013) en Tanzanie qui ont montré que la disponibilité de plusieurs hôtes fait que $B$. dorsalis est présente pendant toute l'année. Steiner et al. (2008); VanDerWal et al. (2009) et Desneux et al. (2010) rajoutent que les conditions environnementales propices favorisent le développement et le maintien de 
B. dorsalis. Dans tous les sites de collecte, les captures sont légèrement plus élevées en saison humide qu'en saison sèche. Cependant, les sites 2 et 4 se distinguent par les captures les plus importantes quelle que soit la saison. Ceci s'explique par l'état sanitaire de ces vergers presque abandonnés.

Dans notre zone d'étude, la présence ou l'absence de fruits hôtes en saison pluvieuse ou sèche semble être une des causes fondamentales de la dynamique des mouches des fruits. Duyck et al. (2004) confirment que la disponibilité de fruits-hôtes affecte à la fois le développement des stades immatures ainsi que le comportement des adultes.

\section{Influence des facteurs climatiques sur la dynamique}

Une relation étroite existe entre la température, la pluviométrie, l'hygrométrie et les captures par sites dans l'espace et dans le temps à l'exception du vent.

L'évolution des captures est bien corrélée à celle de la température ambiante. La température a une influence très importante sur le développement et la survie des Tephritidae (Duyck, 2010). Les fortes captures sont observées durant les périodes les plus chaudes allant de mai à novembre et les faibles captures pendant les périodes plus froides. Ce résultat confirme les travaux de Vargas et al. (2000), Brévault et Quilici (2000) et Duyck et Quilici (2001) qui ont montré que la survie (plus de 75\%) de tous les stades de mouches des fruits notamment de $C$. capitata est élevée dans la gamme de température $15-30{ }^{\circ} \mathrm{C}$. Ces mêmes auteurs ont montré que le temps de développement de l'œuf à l'adulte de $C$. capitata se situe entre 16 et 64 jours, respectivement pour les températures de 30 et $15^{\circ} \mathrm{C}$. Rwomushana et al. (2008b) rajoutent pour B. dorsalis qu'en Afrique, les seuils inférieurs de développement pour les œufs, les larves et les nymphes sont respectivement de 8,$8 ; 9,4$ et $8,7^{\circ} \mathrm{C}$. Selon Stephens et al. (2007). $42{ }^{\circ} \mathrm{C}$ est la température limite maximale de développement de $B$. dorsalis et que l'optimale est obtenue à $33{ }^{\circ} \mathrm{C}$. Les plus importantes captures par piégeage sont obtenues à la température de $40{ }^{\circ} \mathrm{C}$ selon le même auteur. La température joue un rôle déterminant dans l'abondance des Tephritidae à travers ses effets sur les taux de développement, de mortalité et de fécondité de ces insectes selon Ouédraogo (2007).

L'analyse de l'influence de l'humidité (hygrométrie et pluviométrie) sur la fluctuation des captures montre également que l'humidité de l'environnement a une influence particulièrement importante dans l'abondance de nombreuses espèces de Tephritidae. La distribution de la mouche des fruits est largement déterminée par l'humidité. En effet, selon Stephens et al. (2007), les limites Nord de la répartition de $B$. dorsalis en Afrique sont liée la sécheresse de la Zone. De Villiers et al. (2015), ont montré également que les principaux facteurs limitant dans la distribution de $B$. dorsalis dans la limite Nord de l'Afrique sont principalement la sécheresse et le froid. Ndiaye et al. (2007) ont montré pareillement l'importance de la quantité de pluies tombées sur le développement de la population de B. dorsalis au Sénégal. Vayssières et al. (2009d) ont montré aussi qu'il existe une corrélation significative positive entre les précipitations et les captures de B. dorsalis dans les vergers du Nord Bénin.

Selon Ouédraogo (2007), l'humidité agit sur l'abondance des populations de Tephritidae à travers la réduction de la fécondité des femelles en période sèche et par la forte mortalité des adultes nouvellement émergés dans des conditions sèches (sol sec et atmosphère à faible humidité relative). Ces résultats se rapprochent également des travaux de Vayssières et al. (2006) qui ont montré que les fluctuations des populations de Bactrocera dorsalis suivent la dynamique des pluies. Sutherst et al. (2007), ont montré pareillement que l'humidité du sol, la température ainsi que la longueur du jour à un effet sur la diapause de B. dorsalis. Jiron et Hedström (1991) et Tan et Serit (1994) rajoutent également que l'humidité a une influence sur les fluctuations 
des populations des mouches des fruits pendant les périodes de fructification de plantes hôtes. Une partie du cycle de développement de l'insecte s'est réalisée dans le fruit et une autre partie dans le sol. La synchronisation de la période de fructification et des facteurs abiotiques (pluviométrie, température, et humidité) est primordiale pour la dynamique de populations de mouches des fruits (Vayssières et al., 2009). Stephens et al. (2007) et l'OEPP (2010) dans le modèle actuel de distribution basée sur la répartition et la dynamique saisonnière de $B$. dorsalis en Afrique, ont montré que l'humidité adéquate pour le développement de la larve de Bactrocera dorsalis dans le sol se situe dans les seuils extrêmes de 0,1 et 1,5. Wih (2008), Vayssières et al. (2009a) et De Meyer et al. (2010) ont également montré qu'en en Afrique, B. dorsalis préfère des niveaux de précipitations et d'humidité relative élevés

En ce qui concerne le vent, une corrélation négative non significative est observée entre les captures par site et la vitesse du vent. Le vent réduit le mouvement de la mouche des fruits. Lorsque la vitesse du vent est importante, les mouches se réfugient dans le feuillage des plantes (Mille, 2010). En outre, les insectes sont désorientés par le vent qui emporte plus loin l'odeur de la phérormone.

Il est à noter également que les jeunes fruits de mangues chutés du fait de la coulure physiologique et la cassure de branches sous l'effet de la charge en fruits au cours du grossissement constituent un important foyer de multiplication de mouches des fruits, causant des dégâts en période de récolte. Les premières précipitations de juin à l'entame de la maturité des mangues sur pieds renforcent les conditions de multiplication des ennemis déjà entamées sur les jeunes fruits chutés et favorisent leur migration des jeunes fruits chutés vers les fruits sur pieds en maturité physiologique. Les pics et les dégâts enregistrés en juillet et août correspondent à la maturité d'une bonne partie des variétés de mangues et à l'émergence de générations de pupes ayant évolué sur les jeunes fruits chutés sous les houppiers des manguiers. Les mêmes résultats ont été signalés par Ali AhmedSadoudi (2007) qui a enregistré un maximum de captures pendant la période qui coïncide avec la maturité des figues.

A partir des résultats obtenus sur l'analyse de la régression multiple, il est noté que seule l'humidité relative permet d'expliquer significativement les captures au cours de la période d'étude. Ces résultats concordent avec ceux de Mwatawala et al. (2006); Vayssières et al. (2008b, 2009d) qui ont montré que $B$. dorsalis est une espèce qui se développe dans des conditions de fortes humidités, la diminution de l'humidité de l'air entraîne une diminution des captures de $B$. dorsalis. Selon Ouedraogo (2011), les faibles valeurs de l'humidité relative moyenne et la rareté des ressources alimentaires, expliquent les faibles abondances de B. invadens aussitôt après la saison des mangues. Pour C. cosyra, Caroll et al. (2002) et Vayssières et al. (2009c) ont montré que les valeurs élevées de l'humidité relative sont défavorables au développement des populations de $C$. cosyra adaptée aux conditions sèches de l'environnement qui caractérisent certaines périodes de l'année. Ouédraogo (2007) a noté qu'une augmentation de l'humidité relative de l'air entraîne une augmentation des attaques de mouches sur la mangue et inversement.

Mille (2010) a montré que les profondeurs de pupaison diffèrent en fonction de l'humidité ainsi que de l'interaction température, type de sol et humidité. Ce même auteur rapporte que la profondeur d'enfouissement des pupes varie selon les espèces et l'humidité sol. Pour Bactrocera dorsalis, Zaoui (2012) a montré qu'elle réalise sa pupaison régulièrement entre 2 et $3 \mathrm{~cm}$ de profondeur sous la frondaison des arbres fruitiers en sol non compact, friable et humide. Sur les agrumes, Tamesse et Messi (2004) ont montré que les valeurs faibles de l'humidité relative entraînent une diminution des effectifs d'individus du psylle (Trioza erytreae). Dans les vergers sud-africains, une 
baisse de l'humidité relative entraîne celle du niveau des populations des psylles. Dans les conditions de verger, les valeurs minimales prolongées de l'humidité relative sont par conséquent préjudiciables à la survie et au développement de $T$. erutreae (Tamesse et Messi, 2004).

\section{Conclusion}

L'observation des résultats des captures mensuelles de $B$. dorsalis entre juillet 2012 et juillet 2014 montre que de façon générale, les captures les plus faibles sont observées en décembre et en mars, et augmentent de mai à juillet. Mais selon l'année, les captures peuvent être différentes. Les captures ont été nettement plus élevées au mois de juillet 2012 dans les 4 sites que tous les autres mois sur les deux ans. En 2013, les captures sont plus élevées en juillet dans les sites 1 et 3 et en août dans les sites 2 et 4 . En 2014, les captures sont plus importantes en juillet dans les sites 1, 2 et 3 et en juin dans le site 4 ; elles sont faibles dans tous les sites en janvier, février et mars. Les captures sont différentes selon les sites et les mois dans l'année.

Entre les deux saisons, les captures ont été plus élevées en saison humide qu'en saison sèche. Malgré les fluctuations constatées, il convient de noter que dans tous les vergers étudiés, $B$. dorsalis est présente, à des degrés différents, presque toute l'année.

Il existe une relation étroite entre les facteurs climatiques notamment la température, la pluviométrie, l'hygrométrie et les captures par sites dans l'espace et dans le temps à l'exception du vent. Mais l'humidité relative semble mieux expliquer l'influence des facteurs climatiques sur la dynamique de mouches des fruits dans les vergers étudiés. Les fluctuations des captures observées au cours de l'étude sont le fait à la fois de l'influence de facteurs climatiques et de la présence ou de l'absence de fruits hôtes. Cependant, il est à préciser que les variations climatiques à elles seules ne peuvent pas expliquer la fluctuation des populations de mouches des fruits dans les vergers étudiés. La charge en fruits, la variété et les stades phénologiques sont aussi responsables de la fluctuation.

A cet effet, la condition préalable à l'élaboration d'une méthode de lutte intégrée contre les ennemis de cultures, repose sur une connaissance approfondie de la dynamique des populations de mouches des fruits. Les périodes de chutes de jeunes fruits de mangues doivent donc être surveillées parce qu'elles permettent une multiplication intense de mouches des fruits sous les manguiers qui occasionnent des dégâts sur les mangues en maturité sur pieds. Des interventions pendant les périodes de faibles fluctuations seraient nécessaires pour lutter contre les dégâts causés par ces ennemis en période de maturité des mangues.

\section{REMERCIEMENTS}

Ce document est lié aux recherches conduites par le laboratoire de Phytochimie et Protection des Végétaux (PPV) du Département de Biologie Végétale de l'Université Cheikh Anta Diop de Dakar (UCAD) et en collaboration avec l'ISRA (Institut Sénégalais de Recherches Agricoles) et le Laboratoire de Zoologie des Invertébrés terrestres, Institut fondamental d'Afrique Noire (IFAN) de Dakar (Sénégal). Nos remerciements sont adressés à l'ensemble de l'équipe de l'ISRA/DJIBELOR et du Laboratoire de Zoologie des Invertébrés terrestres de l'IFAN pour son soutien pendant les travaux de terrain.

\section{CONFLIT D'INTERET}

Les auteurs déclarent qu'ils n'ont aucun conflit d'intérêts.

\section{CONTRIBUTIONS DES AUTEURS}

ISK a été l'investigateur principal ; SD a contribué à la collecte des données et à la rédaction du manuscrit; SS a contribué à la rédaction du manuscrit; $\mathrm{LD}$ a contribué à la collecte des données; $\mathrm{ABN}$ et $\mathrm{KN}$ ont 
contribué à la conception du sujet et rédaction du manuscrit.

\section{REFERENCES}

Ali Ahmed-Sadoudi D. 2007. Bio écologie de la mouche méditerranéenne des fruits Ceratitis capitata Wiedemann, 1824 (Diptera : Trypetidae) dans quelques vergers de la Kabylie. Thèse de Doctorat de l’Université de Tizi-Ouzou, p. 197.

Ali Ahmed-Sadoudi D, Metna F, Kherroubi S. 2014. Dynamique des populations de ceratitis capitata wiedemann (diptera, tephritidae) sur la variété d'orange thomson dans différents vergers d'agrumes de la kabylie. Deuxième conférence internationale sur les ravageurs en agriculture, Montpellier - 22 et 23 octobre 2014 , p. 8 .

Asepex. 2012. Bilan de la campagne 2012 d'exportation mangue au Sénégal. Agence Sénégalaise de Promotion des Exportations. Sicap Amitié 3, n4426-BP 14709 Dakar Peytavin, Sénégal. Étude mangue. Rapport de la cellule d'information et de veille commerciale $\mathrm{p}$. 4.

Ba CO. 2004. Migrations et organisations paysannes en Basse Casamance. Cas du village de Suel, département de Bignona, ISRA-Djibélor, Sénégal : p. 80.

Brévault T, Quilici S. 2000. Relationships between temperature, development and survival of different life stages of the tomato fruit fly, Neoceratitis cyanescens. Entomologia Experimentalis et Applicata, 94: $25-30$.

Carroll LE, White IM, Freidberg A, Norrbom, AL, Dallwitz MJ, Thompson FC. 2002. Pest Fruit Flies of the World: Identification, Descriptions, Illustrations, and Information Retrieval, USDA-ARS. Available online: http://www.sel.barc.usda.gov/Diptera/tep hriti/pests/adults/

De Meyer M, Robertson MP, Mansell MW, Ekesi S, Tsuruta K, Mwaiko W, Vayssières J-F, Peterson AT. 2010.
Ecological niche and potential geographic distribution of the invasive fruit fly Bactrocera invadens (Diptera, Tephritidae). Bulletin of Entomological Research, 100: 35-48.

De Villiers M, Hattingh V, Kriticos DJ, Brunel S, Vayssières J-F, Sinzogan A, Billah MK, Mohamed SA, Mwatawala M, Abdelgader H, Salah FEE, De Meyer M. 2015. The potential distribution of Bactrocera dorsalis: considering phenology and irrigation patterns. Cambridge University, Bulletin of Entomological Research, 1-15.

Desneux N, wajnberg E, wyckhuys KAG, Burgio G, Arpaia S, Narvárez-Vasquez CA, González-Cabrera J, Catalán Rruescas D, Ttabone E, Frandon J, Pizzol J, Poncet C, Cabello T, Urbaneja A. 2010. Biological invasion of European tomato crops by Tuta absoluta: ecology, geographic expansion and prospects for biological control. Journal of Pest Science, 83: 197-215.

Dieng M, Diop EO, Diatta H, Gaye M, Delhoven M, Dieng GB. 2007. Suivi des populations des mouches des fruits et démonstration d'un dispositif de lutte intégrée dans des vergers de manguiers. Direction de la protection des végétaux, rapport, p.15.

Duyck PF, Quilici S. 2001. Etude comparée de la biologie du développement chez trois espèces de mouches des fruits (ceratitis spp.) (Diptera : Tephritidae), nuisibles aux cultures fruitières a la réunion. Food and Agricultural Research Council, Réduit, Mauritius, p. 9.

Duyck P-F, David P, Quilici S. 2004. A review of relationships between interspecific competition and invasions in fruit flies (Diptera: Tephritidae). Ecological Entomology, 29: 511-520.

EPPO (2010) Pest risk analysis for Bactrocera invadens. Available online at http://www.eppo.int/QUARANTINE/Pest _Risk_ Analysis/PRA_intro.htm (accessed May 2014). 
Guerts k, Mwatawala M, De Meyer M. 2013. Indigenous and invasive fruit fly diversity along an altitudinal transect in Eastern Central Tanzania. Journal of Insect Science, 12: 12. Available online at: insectscience.org/12.12

Heger T, Trepl L. 2003. Predicting biological invasions. Biological Invasions 5: 313321. Information Retrieval, USDA-ARS. Available online.

Magagula CN, Cugala DC, Monadjem A, Dlamini WM. 2015. Predicted Regional and National Distribution of Bactrocera dorsalis (syn. B. invadens) (Diptera: Tephritidae) in Southern Africa and Implications for Its Management. African Entomology, 23(2): 427-437.

Mané A. 2008. Plantes hôtes de Bactrocera invadens Drew (Diptera Tephritidae) en Casamance au Sénégal. Mémoire de fin d'étude pour l'obtention du Diplôme d'Ingénieur de l'Institut Polytechnique Rural de Formation et de Recherche Appliquée (IPR/IFRA) de Katibougou, p. 91.

Mille C. 2010. Les mouches des fruits de Nouvelle-caledonie (diptera, tephritidae) : Systématique, Comportement, Dynamique et Gestion des Populations. Physiologie et Biologie des Organismes. Thèse de Doctorat, école doctorale du pacifique, p.353.

Mwatawala MW, de Meyer M, Makundi RH, Maerere AP. 2006a. Biodiversity of fruit flies (Diptera, Tephritidae) in orchards in different agro-ecological zones of the Morogoro region. Tanzania Fruits, 61(5): 321-332.

Ndiaye M, Lessueur. S. 2012. The effectiveness and attractiveness of the trap "Sentrap" and two home-made prototypes to "Multilure" (conventional trap) in male anihilation technique. African Journal of Biotechnology, 11(34): 4.

Ndiaye O. 2014. Interactions plantes hôtes, mouches des fruits et parasitoïdes dans la zone des Niayes et en Casamance. Thèse de Doctorat de l'Université Cheikh Anta Diop de Dakar/ Sénégal. p.162.

Ndiaye M, LÔ MN. 2008. Etude et analyse des différentes initiatives en vue d'une stratégie régionale de lutte contre les mouches des fruits en Afrique de l'ouest et du centre. Conférence des Ministres de l'Afrique de l'Ouest et du centre. CMA/AOC. p. 19.

Ouédraogo SN, 2007. Etude des attaques des mouches des fruits (Diptera: Tephritidae) sur la mangue dans la province du Kénédougou (Ouest du Burkina Faso). Mémoire de DEA, Institut du Développement Rural, Université Polytechnique de Bobo Dioulasso, 58 p.

Rwomushana I, Ekesi S, Ogol CKPO, Gordon I. 2008b Effect of temperature on development and survival of immature stages of Bactrocera invadens (Diptera: Tephritidae). Journal of Applied Entomology, 132: 832-839.

Sonkho Y. 2010. Évaluation de la dynamique de populations de Bactrocera invadens (Ddrew Ttsuruta \& Wwhite) et de Ceratitis cosyra (walker) (Ddiptère Ttephritidae) dans les zones de production de mangues du département de Ziguinchor. Mémoire de fin d'études pour l'obtention du diplôme d'ingénieur des travaux, p. 66.

Steiner FM, Schlick-Steiner BC, Vanderwal J, Reuther KD, Christian E, Stauffer C,

Suarez AV, Williams SE, Crozier RH. 2008. Combined modelling of distribution and niche in invasion biology: a case study of two invasive Tetramorium ant species. Diversity and Distributions, 14: 538-545.

Stephens AEA, Kriticos DJ, Leriche A. 2007. The current and future potential geographical distribution of the oriental fruit fly, Bactrocera dorsalis (Diptera, Tephritidae). Bulletin of Entomological Research, 97: 369-378.

Sutherst RW, Maywald GF. 1985. A computerised system for matching climates in ecology. Agriculture, 
Ecosystems and Environment, 13: 281299.

USDA. 2011. Nutrient Database for Stanadrd Référence in Djioua. Thèse de doctorat, Université paris Est. Ecole doctorale science de la vie et de la sante, p. 194.

VanDerWal J, Shoo LP, Johnson CN, Williams SE. 2009. Abundance and the environmental niche: environmental suitability estimated from niche models predicts the upper limit of local abundance. The American Naturalizt, 174(2): 282-291.

Vargas RI, Walsh WA, Kanehisa D, Stark JD, Nishida T. 2000. Comparative demography of three Hawaiian fruit flies (Diptera : Tephritidae) at alternating temperatures. Annals of the Entomological Society of America, 93: 75-81.

Vayssières JF, Sinzogan AAC, Korie S, Ouagoussounon I, Thomas-Odjo A. 2009b. Effectiveness of spinosad bait sprays (GF-120) in controlling mangoinfesting fruit flies (Diptera: Tephritidae) in Benin. Journal of Economic Entomology, 102 (2): 515-521.

Vayssières J-F, Korie S, Ayegnon D. 2009a. Correlation of fruit fly (Diptera: Tephritidae) infestation of major mango cultivars in Borgou (Benin) with abiotic and biotic factors and assessment of damage. Crop Protection, 28: 477-488.

Vayssières J-F, Korie S, Ayegnon D. 2009d. Correlation of fruit fly (Diptera
Tephritidae) infestation of major mango cultivars in Borgou (Benin) with abiotic and biotic factors and assessment of damage. Crop Protection, 28: 477- 488.

Vayssieres J-F, Sinzogan A, Bokonon-Ganta A. 2008b. La nouvelle espèce invasive de mouche des fruits : Bactrocera invadens Drew Tsuruta \& White. Fiche technique 2, CIRAD, UPR Production Fruitière, Montpellier, France; IITA Cotonou, 4 pp.

Vayssières JF, Lokosou O, Ayégnon D, Bouéyi SP, Akponon C. 2006. Inventaire, fluctuations des populations et importance des dégâts des espèces de mouches des fruits (Dipteria Tephritidae) inféodés au manguier dans le département duBorgou (Bénin). In INRAB. Atelier scientifique national de l'INRAB, du 19 au 22 décembre 2006, Cotonou, Bénin.

Wih K. 2008. Assessment of fruit fly damage and implications for the dissemination of management practices for mango production in the upper west region of Ghana. Journal of Developments in Sustainable Agriculture, 3: 117-134.

Zaoui R, Zine S. 2012. Effet de quelques paramètres abiotiques sur la phase hypogée de Bactrocera oleae (Diptera : Tephritidae) dans l'oliveraie de la station régionale e protection des végétaux d'Ain-Touta. Mémoire Master en Sciences de l'environnement. Université de Batna, 53pp. 